\title{
Flow Boiling Numerical Solution through a Water Based Nano-Fluidic Mixture Free Convection
}

\author{
Yasir M. Shariff \\ Mechanical Engineering Department, College of Engineering, Taibah University, Madina, Saudi Arabia \\ Email:yshariff@taibahu.edu.sa
}

How to cite this paper: Shariff, Y.M. (2018) Flow Boiling Numerical Solution through a Water Based Nano-Fluidic Mixture Free Convection. Engineering, 10, 1-6. https://doi.org/10.4236/eng.2018.101001

Received: December 31, 2017

Accepted: January 21, 2018

Published: January 24, 2018

Copyright $\odot 2018$ by author and Scientific Research Publishing Inc. This work is licensed under the Creative Commons Attribution International License (CC BY 4.0).

http://creativecommons.org/licenses/by/4.0/

\begin{abstract}
In this study, a 2-D MHD free convection incompressible electrically induced boundary layer analysis on a water/nano-fluidic mixture. The ODE solution is numerically analyzed with a Runge-Kutta model as the thermophysical properties on a magnetic variation as well as temperature ranges of buoyancy effects on the $\mathrm{T}$ and $\mathrm{V}$ profiles and the wall force friction on the flow is also studied. The temperature and velocity gradients have a significant differential change is been observed in this study.
\end{abstract}

\section{Keywords}

Flow, Boiling, Nano-Fluids, Convection

\section{Introduction}

The device designs have been developed recently specially for moving surfaces in fluid flow over different ranges of geometry and magnetic/electrical effects. Many researchers worked on the moving sheet flow boiling and numerical analysis with solutions for the governing equations of heat and mass transfer [1]. It is found through some of the mentioned studies that $\mathrm{V}$ ratios and other parameters influenced heat transfer results. Other scientists studied the effect of the moving sheets on both flow boiling and its thermal variation in comparison to the flow entrance and exits [2]. The buoyancy forces along the flow values of Re in conjunction with the Gr values as well as Pr values were analyzed on the thermal physical variation effects on the magnetic influence over different mixtures in evaluation of the thermal conductivity on a laminar incompressible flow over a moving sheet of aluminum [3].

Other studies showed that the shear stress of the wall increased with increase 
of flow rates and the effect of MHD boundary layer on the flow with a moving sheet of aluminum with different flow velocity parameters [4]. The laminar BL flow of a water/nano-fluidic mixture over a semi-infinite surface has been studied and analyzed for a free convection effect on an aluminum moving surface [5]. The results showed that the moving surface on a flow characteristic was dependent on the buoyancy effects over the laminar BL and the free convection effect on the surface contact with the mixture versus other research work where the aluminum surfaces moved in the opposite direction [6]. It was found through these studies that the free convection had effects over the moving surface with also heat transfer radiation effect as well but, yet the free convection effects had a better yielding result then compared to forced convection [7]. The present study investigates the magnetic effect and the T/buoyancy variations on the free convection variables with a moving aluminum surface with a water/nano-fluidic mixture [8].

M. M. Sarafraz also investigated the flow boiling parameters in conjunction with the heat characteristics of metal oxide on an array of nanotubes made of carbon and the use of nano-fluids in annulus heat exchanger. Variations of different heat transfer conditions were done experimentally to evaluate the HTC and thermal fouling resistance in conjunction with different heat fluxes, mass concentrations of the nano-fluids. He also prepared the nano-fluids, multiwalled carbon nanotubes, alumina and copper oxide nanoparticles were dispersed into deionized water. The thermal conductivity was measured and taken against published work and it resulted in an increase of its thermal conductivity and boiling thermal performance and a decrease of the fouling resistance as compared to other nano-fluids. Similarly, the boiling performance for the nanofluids in the array of carbon tubes was found intensified when the concentrations of the mixture was increased, finally the experimental htc was verified against other published correlations [9].

Nucleate pool boiling HTC were measured during pool boiling of the mixtures of Citric acid/water on a horizontal heated Cylinder by M. M. Sarafraz et al. Results were found for the pool boiling HTC at different flow parameters with mixtures of citric acid water and available correlations were compared and some of them were not exactly compared well as based model on Stephan-Kroner were found to be verified, furthermore, the bubble growth showed that nucleation density is a function of heat flux [10].

Salari et al. also worked on the potential application of nano-fluids as a cooling agent of high heat flux surfaces. Their work was based on the experimentation of the HTC of Titana aqueous nano-fluids against variations of temperature and heat/mass fluxes. Variant fluxes of heat/mass concentrations of the nano-fluids were studied for the boiling characteristics on a copper surface at different subcooled T. It was found that the HTC increases as compared to the base fluid as well the heat/mass concentrations of the nano-particles can only affect the pool boiling HTC at subcooled T can also affect the bubble growth. Salari et 
al. observed that fouling formation of the nano-particles has affected the intensity of the bubble dynamics due to the intensity of nucleation sites in the boiling surface [11].

\section{Results and Discussion}

The flow parameters and its analysis is given in the solution through defining the figure model of the system that deals with a 2-D MHD boundary layer, electrically conductive viscous constant density assumption through the flow boiling condition with continuity, momentum, and energy equation solution of the first order laminar flow condition, but in this particular analysis the nonlinear ordinary differential equation will be guided through the assumed ignition conditions with an adaption of the Runge-Kutta model as this technique provides discretizing of the solution for the variable BC. Large values are effective of the boundary conditions with a solution for the domain which satisfies the solution type expected for a 2-D model. Furthermore, stability and step size iteration solution model were consistent and for the solution of the nonlinear equations it is transformed into $1^{\text {st }}$ ODE as there are 3 conditions for the boundary to define the variable initial conditions. For the adaptive solution method for the RK is taken with an iteration to account for the error control on the adjustment increments which approximately at an initial step size of h equals to 0.04 .

A computational solution is presented in Figure 1, for the concentrations, velocities, and the shear forces on the flow gradients against constant values of the

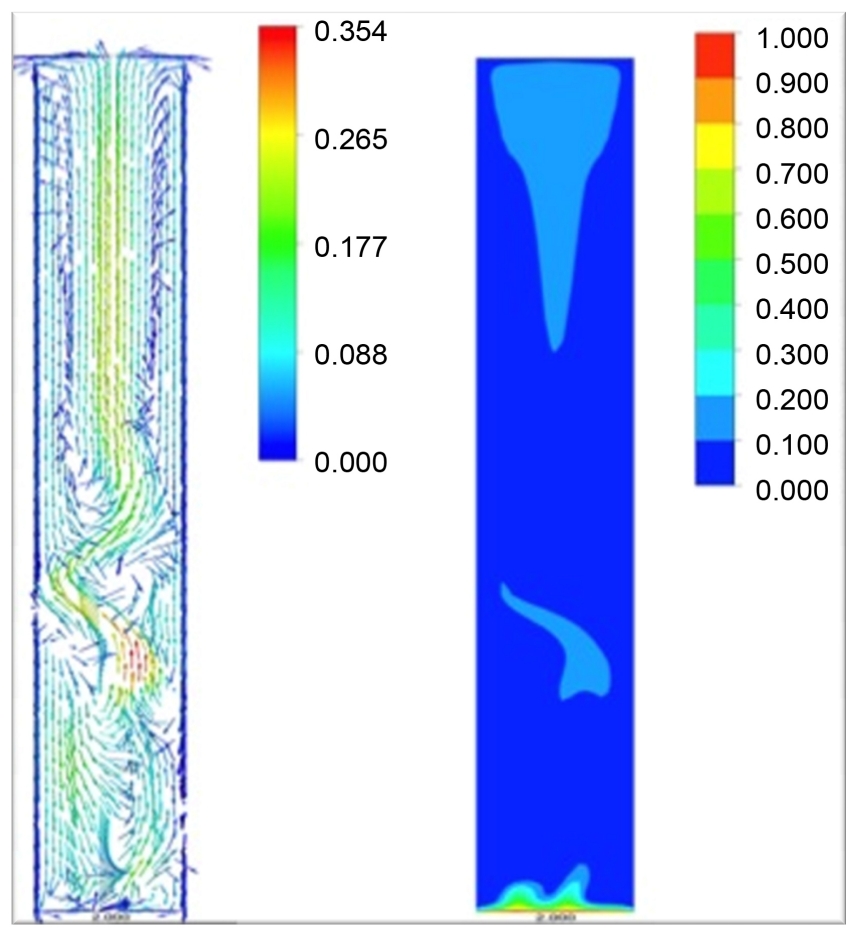

Figure 1. Thermal effects with different flow boiling parameters over a vertical flow contours with gradients of temperature currents along the wall with the RK model. 
magnetic dimensions as well as the operation temperature of the buoyancy at $\mathrm{Pr}$ number greater than 7 with the $\mathrm{Al} /$ water nano-fluidic mixture at an ambient temperature of 30 degrees $\mathrm{C}$. The variable considered has been modeled to presents the $\mathrm{T}$ of buoyancy against the flow boiling measures with different profile concentrations and through controlling the velocity into a decrease as well as the shear forces with an increase of the magnetic values.

Both the magnetic effects on the flow and temperatures were increased with a gradient and were evaluated with a decrease of the magnetic effects with incremental values as it showed an effect on the velocity of the flow against the natural convective values as well as the shear forces and the concentration values. It is found that the velocity increases with increase of natural convective currents and the same can be observed through the shear forces as is demonstrated in

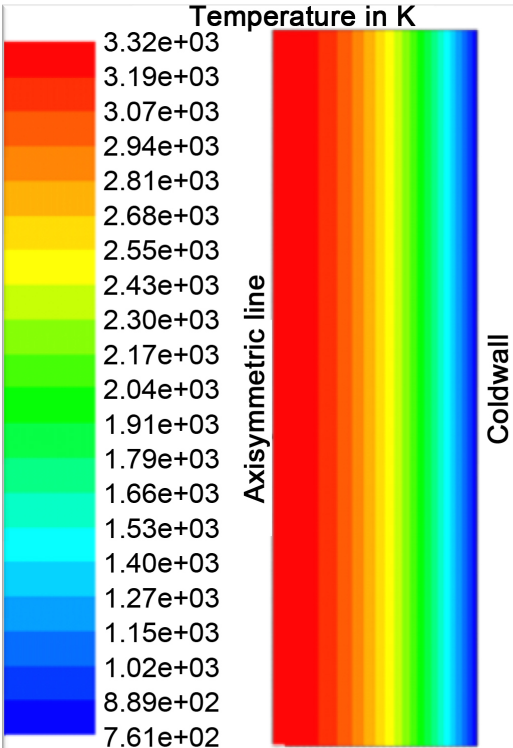

(a)

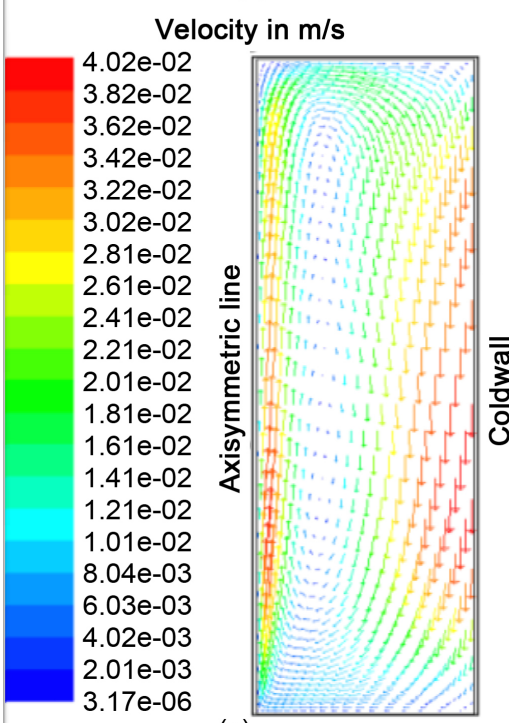

(c)

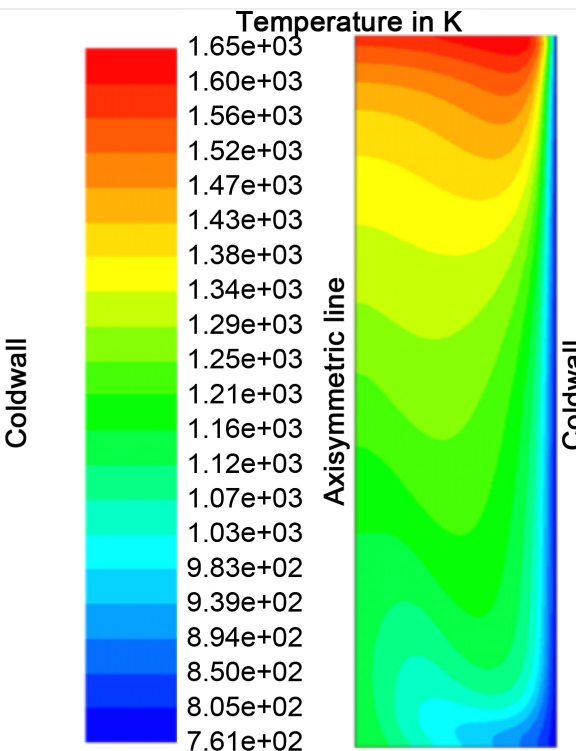

(b)

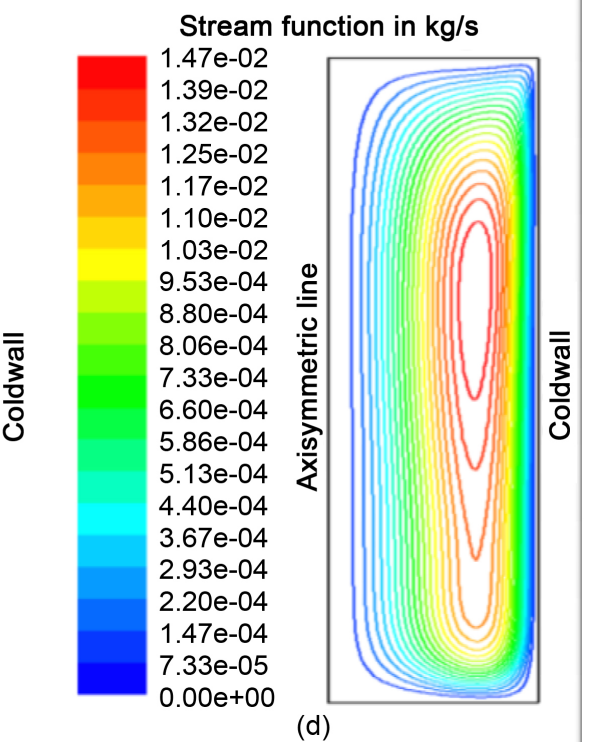

Figure 2. The effect of the natural convection gradient on the wall temperatures. 
Figure 2. Therefore, the temperature ranges against the convective profiles were also observed as it is found that the temperatures significantly increased with increase of the natural convective effects such as buoyancy.

\section{Conclusion}

A derived aluminum surface with various velocities against magnetic and natural convective effect on a nano-fluidic flow boiling is presented as the nonlinear PDE are solved to present a nonlinear ODE model using similarity manipulation and finding a solution numerically by the RK $5^{\text {th }}$ order of the frictional coefficients and the shear forces. It is found that increasing the magnitude of the magnetic effects decrease both $\mathrm{V}$ and the shear forces against the wall and brings and enhancing effect on friction and temperatures. The shear forces in the other hand are affected against both the temperature and magnetic gradients. The temperatures of the natural convection effects increase with increase of the flow parameters and the shear force on the wall and the friction coefficient and temperature variables decreased.

\section{Acknowledgements}

This work was supported by the National Science, Technology and Innovation Plan (NSTIP) through the Science and Technology Unit (STU) at Taibah University, Al Madinah Al Munawwarah, KSA, with the grant number 08-NAN20-5.

\section{References}

[1] Zheng, L.C. and Zhang, X.X. (2002) Skin Friction and Heat Transfer in Power-Law Fluid Laminar Boundary Layer along a Moving Surface. International Journal of Heat and Mass Transfer, 45, 2667-2672. https://doi.org/10.1016/S0017-9310(01)00363-5

[2] Al-Sanea, S.A. (2003) Convection Regimes and Heat Transfer Characteristics along a Continuously Moving Heated Vertical Plate. International Journal of Heat and Fluid Flow, 24, 888-901. https://doi.org/10.1016/j.ijheatfluidflow.2003.10.001

[3] Seddeek, M.A. (2003) Flow of a Magneto-Micropolar Fluid past a Continuously Moving Plate. Physics Letters, Section A: General, Atomic and Solid State Physics, 306, 255-257. https://doi.org/10.1016/S0375-9601(02)01513-X

[4] Patel, H.E., Sundararajan, T., Pradeep, T., Dasgupta, A., Dasgupta, N. and Das, S.K. (2005) A Micro-Convection Model for Thermal Conductivity of Nanofluids. Pramana-Journal of Physics, 65, 863-869.

[5] Cortell, R. (2007) Flow and Heat Transfer in a Moving Fluid over a Moving Flat Surface. Theoretical and Computational Fluid Dynamics, 21, 435-446. https://doi.org/10.1007/s00162-007-0056-Z

[6] Ishak, A., Nazar, R. and Pop, I. (2008) MHD Boundary-Layer Flow Due to a Moving Extensible Surface. Journal of Engineering Mathematics, 62, 23-33. https://doi.org/10.1007/s10665-007-9169-z

[7] Bachok, N., Ishak, A. and Pop, I. (2010) Boundary-Layer Flow of Nanofluids over a Moving Surface in a Flowing Fluid. International Journal of Thermal Sciences, 49, 1663-1668. https://doi.org/10.1016/j.ijthermalsci.2010.01.026

[8] Habib, H.M. and El-Zahar, E.R. (2013) Mathematical Modeling of Heat-Transfer 
for a Moving Sheet in a Moving Fluid. Journal of Applied Fluid Mechanics, 6, 369373.

[9] Sarafraz, M.M. and Hormozi, F. (2016) Comparatively Experimental Study on the Boiling Thermal Performance of Metal Oxide and Multi-Walled Carbon Nanotube Nanofluids. Powder Technology, 287, 412-430.

https://doi.org/10.1016/j.powtec.2015.10.022

[10] Sarafraz, M.M. (2012) Nucleate Pool Boiling of Aqueous Solution of Citric Acid on a Smoothed Horizontal Cylinder. Heat and Mass Transfer, 48, 611-619.

[11] Salari, E., Peyghambarzadeh, S.M., Sarafraz, M.M. and Hormozi, F. (2016) Boiling Thermal Performance of $\mathrm{TiO}_{2}$ Aqueous NanoFluids as a Coolant on a Disc Copper Block. Periodica Polytechnica Chemical Engineering, 60, 106-122. 